\title{
Discussion
}

\section{Alzheimer research forum live discussion: Memantine: Implications for treating Alzheimer's ${ }^{1}$}

Steven T. DeKosky and Bengt Winblad led this live discussion on 10 February 2004. This discussion was co-organized by the Alzheimer's Association and the Alzheimer Research Forum.

http://www.alzforum.org/res/for/journal/dekosky/default.asp

Participants: Antonio Alvarez (caregiver), Steven T. DeKosky (University of Pittsburgh School of Medicine), Mike Faux (caregiver), Dale Grillot (caregiver), Kevin Foley (Hauenstein Alzheimer's Disease and Memory Disorders Program, Grand Rapids, Michigan), Bev Jones (Clinical Associate Professor, Wake Forrest University, Geriatric Psychiatrist), Jim Kallio (caregiver), Zaven Khachaturian (Khachaturian, Radebaugh \& Associates (KRA), Inc.), June Kinoshita (Executive Editor, Alzheimer Research Forum), Pallavi Kakulavar, MD (Cleveland Clinic Foundation), John C. Morris (Washington University), Jeff Paley, MD (Access Medical Associates), Susan Scruby (caregiver), Gabrielle Strobel (Managing Editor, Alzheimer Research Forum), Lon Schneider (University of Southern California), Bengt Winblad (Karolinska Institute, Sweden) Debra Katt-Lloyd (Alzheimer's Association, Massachusetts Chapter), Kim (caregiver).

June Kinoshita: Thank you all for logging on today. Let us begin.

Bengt Winblad: I do think we agree that we have evidence enough to believe that treatment of the disturbances in the glutamatergic system is important in AD.

June Kinoshita: I would like to pose a question to Bengt. Can you give us a general description of the type of patient who you find tends to benefit from memantine?

Bengt Winblad: My feeling is that most of our AD patients will respond on memantine. Certainly, we have the most experience with patients who are in a

\footnotetext{
${ }^{1}$ Note: The transcript has been edited for clarity and accuracy.
}

more advanced dementia stage. We see a rather quick response within two to three weeks, and that is very positive.

Steve DeKosky: Bengt, if you could add ... What is the most likely signal of response seen by you and your staff?

John C. Morris: Bengt, what are the responses not only that you detect, but also that the families report?

Bengt Winblad: The earliest signs are that the patients take more interest in what is around them and take part in activities they have not done before. Examples could be that severely ill patients can start to eat by themselves, which they have not done for a long time.

June Kinoshita: What about effects on language and other types of cognitive functioning? 
Bengt Winblad: Caregivers and family members say that their wives or husbands are more interactive, want to discuss more, take more initiatives. For example, one husband who is a taxi driver told that when he came home, his wife had been out shoveling the snow away from the garage entrance, which she had not done for a long time.

Jim Kallio: Are we assuming in this discussion that Namenda ${ }^{\circledR}$ is being used in conjunction with a cholinesterase inhibitor, too?

Steve DeKosky: The experience of the nursing home study was without cholinesterase inhibitors. Bengt, I assume your comment was for memantine alone?

Bengt Winblad: Yes, I have commented only for monotherapy.

Bev Jones: I see many patients in AD special care units whose Mini-Mental State Examination (MMSE) is < 5. Recently, another physician has started several such residents on Namenda ${ }^{\circledR}$. Is it reasonable to expect a response in these severely affected patients?

Steve DeKosky: Bev, there will obviously be quite impaired people, non-mobile, perhaps with contractures, on whom determining a good response would be difficult. Much of the problem in designing these studies, and the reason that the early studies with cholinesterase inhibitors were done with mild to moderate patients, was because there was belief that it would be easier to show a positive effect on milder patients, and because there were no good tools to assess more severe cases for improvement or stabilization. We are now doing better in that regard.

Bengt Winblad: Bev, in our Latvian study we had a positive effect, also, on these patients. I have not initiated actual patients in nursing homes during the last year.

Bev Jones: Thanks. I realize data is limited on the most severely affected patients, but many families will be facing the question of whether to try Namenda ${ }^{\circledR}$.

June Kinoshita: Bengt, would you characterize the effects of memantine as being an overall improvement across the spectrum of symptoms, or is it more effective for certain aspects of the disease?
Bengt Winblad: June, I believe, without proof, that it has a good effect over the whole continuum.

Zaven Khachaturian: Bengt (Steve, Lon, and John, also), how much experience is there with this drug in the earliest stages of the disease (mild or even earlier)? Given the fact that the mode of action is through the glutamatergic system, one would expect it might be useful slowing disease progression by reducing putative calcium toxicity.

Bengt Winblad: Zaven, I agree about the possibility of disease modification. The preclinical data presented on memantine and its neuroprotective effects are really good and interesting.

I suppose we all have seen the new data on memantine with a possible effect also in mild and moderate stages of $\mathrm{AD}$. Will that mean that we can treat the whole continuum of the disease?

Lon Schneider: The companies did not release any data, just their news releases. I do not think any of us have been able to evaluate the data. Lundbeck's trial was not positive.

Bengt Winblad: Lon, we all agree that our authorities will demand two pivotal studies that are positive. I have also seen a press release.

John C. Morris: I am interested in what data are available to support benefits for memantine combined with cholinesterase inhibitors in patients with mild AD; does anyone know about such results?

Bengt Winblad: John, we have only $8-10$ patients with combination. It seems to work well, but in two patients we have seen a confusional reaction that went away when dosage was reduced from 20 to $15 \mathrm{mg}$.

Lon Schneider: John, I think use of the term "combined with" acetylcholinesterase inhibitors is incorrect. The studies have only assessed the effect of memantine in patients who had been taking an AChEI for at least six months. This is an "add-on", not a combination.

Steve DeKosky: The Tariot et al. paper [1] is the only published add-on study with AChE inhibitors and memantine. Other data have been reported in short communications but not yet published and peer-reviewed.

Lon Schneider: Steve, it is notable that in the moderate to severe stage, add-on patients had been on donepezil 
an average of 2.25 years before being randomized to memantine. They benefited from memantine, but the "placebo group" - those on donepezil alone - continued to deteriorate at a rather rapid rate, as though the donepezil was having no effect.

Bengt Winblad: I agree that it could be an effect of memantine alone in the Tariot paper.

Steve DeKosky: Well, it may be unfair to say that donepezil has no effect unless you removed it and saw a further decline.

Gabrielle Strobel: Lon, do you mean to say the donepezil was no longer working in the Tariot et al. patients, and it was not really a combination therapy trial?

Lon Schneider: Gabrielle, a combination trial would randomize patients to donepezil alone, memantine alone, the combination of donepezil and memantine, and placebo.

June Kinoshita: So, Lon, do these data suggest that memantine has an effect on progression?

Lon Schneider: The studies have not been done to assess progression. It is just that memantine has a clinical effect over six months in moderate to severe patients who had been on donepezil for 2.25 years.

June Kinoshita: Are there ongoing trials that can answer the question regarding progression?

Bengt Winblad: June, not that I know about.

Steve DeKosky: As for disease modification, that seems a plausible hypothesis. It awaits a disease modification study, of longer duration, to see if there is improvement. If you look at the article on neuroprotection in Parkinson's in JAMA last week by Schapira and Olanow [2], you can see what the problems are of interpreting such studies when there is also a symptomatic effect of the drug, as there clearly was with memantine. I am not aware of such a longitudinal study underway. Lon, do you know?

Lon Schneider: Steve, I am not aware of long-term trials.

June Kinoshita: Zaven asked about trials in mild cognitive impairment (MCI) or early AD patients. Are there any in progress?
Bengt Winblad: Zaven, I do not think they have started on MCI yet.

Jim Kallio: We get many questions on the Alzheimer's list about memantine use with Exelon ${ }^{\circledR}$ and Reminyl ${ }^{\circledR}$ ... Will we see trials on those, too?

Antonio Alvarez: This question is for Dr. DeKosky or other doctors in the panel: Are there any data or do you have any opinion on the use of antiinflammatories together with memantine and acetylcholinesterase inhibitors? And specifically, do you think there will be any benefits on the use of Flurbiprofen ${ }^{\circledR}$ as an antiinflammatory medicine together with memantine and an acetylcholinesterase inhibitor? My wife is in the early-mild stages of Alzheimer's.

Steve DeKosky: Antonio, the only treatment trial of use of antiinflammatories in AD (Naprosyn ${ }^{\circledR}$ and ibuprofen) was not positive. There are no data yet in humans on Flurbiprofen, which people are interested in because of its potential effects on amyloid- $\beta$.

Lon Schneider: The R-enantiomer of Flurbiprofen is a non-steroidal antiinflammatory drug (NSAID) but does not inhibit COX1 or 2. It might modulate transcription pathways of $\mathrm{NF} \beta \mathrm{B}$, and it reduces $\mathrm{A} \beta 42$. Myriad Pharma is developing it, but there is no evidence that the use of antiinflammatories is helpful in people who already have AD. In fact, it appears harmful overall.

Jeff Paley: Steve and Bengt, are you believers in the metal-chelation hypothesis and Bush/Tanzi's clioquinol study [3] published recently in Archives of Neurology?

Bengt Winblad: Jeff, clioquinol is difficult to give; you have to add vitamins to avoid side effects. I had an earlier question about the experience of increasing dosage over $20 \mathrm{mg} / \mathrm{day}$. My answer was that there is certainly some experience with memantine in trials on pain with $40 \mathrm{mg} / \mathrm{day}$, but that there is a real risk that higher doses in dementia patients will lead to more side effects. Have you any experiences with higher doses?

June Kinoshita: Thanks for bringing this question up, Bengt. We posted a question before the chat from a woman whose husband seemed to benefit from a higher dose $(40 \mathrm{mg})$, but her physician does not want to go above $20 \mathrm{mg}$. 
Jeff Paley: To all, what does the 10-plus-year experience in Europe tell us about long-term use of memantine?

Bengt Winblad: Jeff, the European experience is that memantine also in the long-term studies is a safe drug and that really few interactions could be expected, and I think that was an important point in the European approval in 2002.

Pallavi Kakulavar: In Europe, are they using memantine for mild Alzheimer's, and if so, do you know if it is effective?

Bengt Winblad: Kakulavar, it is only registered for more severe stages, but mainly in Germany it has been used for several years in milder stages of brain insufficiency, and sold very well.

Pallavi Kakulavar: You mean in Europe it is only approved for use for severe-stage Alzheimer's?

Steve DeKosky: Please remember, everyone, that when you ask whether a medicine is "effective", that the standard is a double-blind, placebo-controlled trial, not our clinical impression ... and there are not a lot of those trials completed and reported.

June Kinoshita: Have any of you had clinical experience treating earlier-stage patients with memantine?

John C. Morris: I have no experience with memantine in early-stage patients. Moreover, I have no experience with memantine in "combination" (or as an add-on) with vitamin E, antiinflammatories, etc. I wonder if there are data from Europe, or if not data, at least clinical experience in this regard?

Bengt Winblad: John, in Europe we do not use vitamin $\mathrm{E}$ in dementia patients and are afraid of antiinflammatories due to the gastric problems. I am sure, still, that Merz would have some data on combinations, but I do not know about them.

Gabrielle Strobel: Bengt, is it not also true that fewer people in Germany take cholinesterase inhibitors? Is memantine the drug of choice over there?

Bengt Winblad: Gabrielle, I think memantine, together with gingko, is having the largest market share in Germany.
Bev Jones: I am interested in the prior European experience, too. For example, was there evidence in other uses that memantine helped moderate to severe disease more clearly than mild?

Kevin Foley: Steve, any trials underway looking at lower dosages or once-daily dosing, or reason beyond the Latvian study to believe that less than $20 \mathrm{mg}$ per day would be just as effective?

Steve DeKosky: Kevin, Bengt would be best to answer your question about lower dosing.

Bengt Winblad: Concerning dosage, I think that for glutamatergic drugs, in general, we believe in a Ushaped dose response curve. Still, $10 \mathrm{mg}$ gave a good effect in our nursing home study.

Jeff Paley: Is there any data from the European literature about use of the drug in severe disease?

Bev Jones: Are there any memantine data on frontotemporal Lewy body disease or Parkinson's?

Bengt Winblad: Bev, I would like to test in a trial for frontotemporal lobe dementia. I have treated three $\mathrm{AD}$ patients with severe frontal lobe atrophy and their reaction was rather modest or bad on the cholinergic drugs, but good on memantine, or actually, the addition of memantine.

Bev Jones: Good! It would seem the benefits would not be specific to AD.

Bengt Winblad: Bev, two European trials on vascular dementia with memantine gave very positive indications of effects $[4,5]$.

Steve DeKosky: There is a great deal of work that has been done in Europe on memantine, and those studies, Bengt's nursing home study, and the two US studies did indeed indicate that memantine appears low in side effects. I think that is why, even with questions about the magnitude of the effect of the medication in terms of changes in behavior or cognition, people are enthusiastic about it and want to try it immediately in milder cases and in other diseases. The clinical experiences will lead to decisions about doing the controlled trials that must be done to demonstrate clearly that it works. Again, the issue of a symptomatic effect versus a neuroprotective effect will complicate the design. 
Bengt Winblad: Steve, I agree.

Dave Grillot: I am a caregiver and researching for a book on caregiving. I have read a number of reports of patients who have become violent when started on memantine. Can anyone comment?

Bengt Winblad: Dave, we have not seen that with monotherapy. I have heard about one case on the combination.

Steve DeKosky: I think there have been some suggestions of agitation in the studies. I do not know of "violence". It is also difficult to characterize what might have led to agitation in many cases of more severe disease. But it deserves continuing monitoring in post-approval assessments of effectiveness.

Lon Schneider: Where do the reports of "violence" come from? Anybody have citations?

Bengt Winblad: Lon, I have seen confusion in two patients, but only heard about more intense psychiatric problems in one.

Dave Grillot: I have seen a number of posts on caregiving messenger boards about families that had to stop memantine because of violent behavior. Sorry, not anything published.

Mike Faux: So is there any data on how long memantine is effective, or what unexpected effects may occur upon withdrawal?

Bengt Winblad: Mike, there is no real long-term data, except that the Barry Reisberg study had an openlabeled phase IV for another 18 months, I believe (personal communication).

Kevin Foley: How does a busy doctor in primary care define "moderate" disease in the office, i.e., a candidate for memantine? Not all doctors use the MMSE or functional scales.

Bengt Winblad: Kevin, I still think that the MMSE is useful on the general practitioner (GP) level. A moderately severe demented patient has a need for support in more complicated tasks, but manages well to live at home.

June Kinoshita: Bengt and Steve, I was struck by the data on adverse events (AEs). In many cases, the memantine group had fewer AEs than the placebo did, which suggests to me that these AEs are actually disease effects that memantine was ameliorating. Would you agree with this interpretation?

Bengt Winblad: June, I think the reduction in, for example, agitation is due to the patient's experience of thinking a bit more clearly; that reduces the problems in the interaction.

Debra Katt-Lloyd: Steve and Bengt, what has been the typical titration in practice? Do you find doctors giving a $5 \mathrm{mg}$ increase per week, or do they tend to spread it out over a longer period, for example, waiting a month in between?

Steve DeKosky: The dose packs have a very slow increase up to $20 \mathrm{mg}$. Most people were starting with 10 , then going to 10 bid when buying the drug from European pharmacies. In the US, the increase has been slower in those packs. But I did not see a problem starting with 10 and going to 10 bid a week later. I am curious about Bengt's greater experience in this regard.

Gabrielle Strobel: Debra, good question. I think for galantamine, the speed with which the doctor ramps up to the full dose affects how well the patient responds and the side effects.

Bengt Winblad: Debra, in practice, I have seen everything going from $10 \mathrm{mg}$ and after one week directly to $20 \mathrm{mg}$. In the patients with frontal lobe engagement, we went much slower than the recommended $5 \mathrm{mg}$ increase per week.

Debra Katt-Lloyd: Thank you, both - my experience with physicians prescribing the cholinesterase inhibitors is often that they would be slow to titrate up even without side effects.

Jeff Paley: Steve and Bengt, is there a downside to adding memantine to acetylcholinesterase inhibitors for all mild to moderate patients, since the safety profile is so clean?

Bengt Winblad: In many of the European countries, the downside would be that there is no reimbursement, and the drugs are certainly very expensive.

Steve DeKosky: In addition to expense, we have a responsibility to figure out how to answer the ques- 
tions in careful ways, such as in the incredibly difficult study Bengt did in Latvia in the nursing home. All our other speculation is just that, and AD patients are, as you know, very variable. The next data that will likely emerge will be from the completed studies of memantine in mild to moderate AD.

Kim: I do not understand. The package insert refers to three studies and the only one with significant results is when memantine is used in combination with donepezil. How can anyone consider using it in monotherapy?

Steve DeKosky: Kim, see Reisberg [6].

Lon Schneider: The package insert refers to three trials, all positive. The Latvia trial had patients with both $\mathrm{AD}$ and vascular dementia.

Kim: What about the Neuropsychiatric Inventory (NPI), Clinician's Interview-Based Impression of Change-plus (CIBIC-plus), and MMSE? Not significant?

Jeff Paley: How might you account for the fact that one study in mild to moderate was negative, but there was a recent one that was positive?

Lon Schneider: NPI was not a primary; the coprimaries included $\mathrm{A} \beta$-derived diffusible ligands (ADDLs).

Bengt Winblad: Lon, a subanalysis of the Latvian study showed that memantine had as good an effect in both Alzheimer's and vascular dementia.

Lon Schneider: Yes, in fact, both the FDA's and Forest Laboratories' subanalyses showed that. I was reacting to the idea that two trials in the package insert were "negative". This is not the case.

Gabrielle Strobel: With cholinesterase inhibitors, clinical experience is exposing an interesting tension between the need to boost ACh levels in the AD brain and to lower them peripherally, for example, to treat incontinence. Anticholinergic drugs are quite commonly prescribed in geriatric care. This can be a dilemma. Do any of you see inklings of a similar mechanistic "tugof-war" for memantine on the horizon, where you want to dampen N-methyl-d-aspartate (NMDA) receptors in $\mathrm{AD}$, but might need to up them for other conditions?
Lon Schneider: Given memantine's action as an NMDA antagonist, you would expect it to reduce the various gastrointestinal symptoms due to acetylcholinesterase inhibitors.

Kevin Foley: Bengt, at what MMSE score would you recommend starting memantine? At 14 ?

Bengt Winblad: Kevin, I would start on patients with MMSE $<15$.

Bev Jones: As a clinician, I expect it will be hard to tell patients and families they should wait until they hit an MMSE $<15$ before starting memantine ...

Jim Kallio: You are right ... Waiting for 15 will be very difficult.

Kevin Foley: So are you going to treat everyone?

Steve DeKosky: Bev, you are right; we all have that dilemma. The issue is how much data you need or trust, and what the expectations are. That is why I think doing the neuroprotection trial is important.

Bengt Winblad: Bev, we can, after the press release from Forest Laboratories, already see that clinicians are using it outside the approved indication on more mild patients.

Jim Kallio: We have the dilemma, too, of when Blue Cross will quit paying for Aricept ${ }^{\circledR}$.

Jeff Paley: Again, Steve or Bengt, any idea why the mild to moderate studies were mixed-some positive and some negative?

Bengt Winblad: Steve, I am not sure a study in MCI will be done, as it is only recognized as an early Alzheimer's diagnosis, and they certainly will have the indications from mild to severe after some further studies.

Steve DeKosky: Bengt, is it likely that Merz or Forest, or both, will do a neuroprotection (long-term) trial to see if it slows progression?

Gabrielle Strobel: On the future outlook: Investigational drugs called cognitive enhancers, or ampakines, modulate synaptic transmission via AMPA receptors, vaguely similar perhaps to the mechanism of meman- 
tine. What do you think of these? Do you foresee future use for $\mathrm{AD}$ from these candidates in the pipeline?

Steve DeKosky: The AMPA receptor is different from the NMDA receptor, and we first need to see positive studies before deciding about any combination therapies aimed at the glutamate receptor subtypes.

Bengt Winblad: I am not so sure that influencing the AMPA receptor is as good an approach, possibly due to more side effects.

Gabrielle Strobel: Bengt, what sorts of side effects?

Bengt Winblad: Gabrielle, side effects more toward the psychotic domain. What is your feeling about the effect in vascular dementia $(\mathrm{VaD})$ ?

Gabrielle Strobel: Steve, you are testing amyloid imaging with Pittsburgh compound B (PIB). Could you do a small study identifying people early and treating them with memantine versus not?

Steve DeKosky: We need to determine what the levels of amyloid are in the brains at different stages of disease, and longitudinally what the rates of increase are in relation to cognition, and then if all goes well, try to see if that intervention slows or reverses amyloid load. I do not think there is a mechanism currently wherein memantine would affect amyloid. But I am sure someone will think of one.

Gabrielle Strobel: Everyone's favorite thing eventually affects amyloid, does it not? Currently, everything is affecting BACE. I thought of it not directly as memantine affecting amyloid, but of presymptomatic amyloid as an early marker.

June Kinoshita: Is there any neuropathology data on patients who have been treated with memantine (and for that matter, AChE inhibitors) regarding effects on plaque load?

Bengt Winblad: June, I am not aware of reduction of plaques. We have run the Pittsburgh PIB ligand in 25 early AD and $20 \mathrm{MCI}$ patients, and in some of these on more occasions, so I am sure Agneta Nordberg will present these data in Philadelphia in July. Steve, in the ECNP meeting there were data presented both on an effect on $\beta$ amyloid production and also on diminishing the phosphorylation of tau in a report from Khalid Iqbal.
Steve DeKosky: It happened already. Very interesting.

June Kinoshita: Bengt, were you addressing your vascular dementia question to anyone in particular?

Bengt Winblad: June, I am happy if anyone will answer if we should do more trials with memantine in vascular dementia.

Lon Schneider: You should distinguish ischemic disease and continue trials in that. That is where the evidence is in Wilcock and Orgogozo's memantine trials $[4,5]$.

Bengt Winblad: Lon, yes, perhaps "vascular dementia" is too broad and heterogeneous.

June Kinoshita: So what would be the inclusion criteria for a vascular dementia trial?

Bengt Winblad: June, probably we have to pick out, as Lon said, a group of patients that on imaging shows a more pronounced subcortical damage indicating that the blood-brain barrier of the penetrating vessels is not intact.

Steve DeKosky: I agree that the cases to focus on may be the subcortical ischemia/lacunar infarction cases ... also easier to track longitudinally, I believe.

Jim Kallio: Yes ... what about using memantine in combination with Exelon or Reminyl?

Steve DeKosky: I suspect the companies are doing those. I do not have a lot of experience with the combination with Exelon; I have a few patients on Reminyl and memantine without problems. But we do very much need a larger experience with these to know that they are okay to use together.

Jim Kallio: How about efficacy, too, on the Exelon and Reminyl used with memantine?

Bengt Winblad: Jim, a large study is ongoing in Germany with a combination with Reminyl. I do think we will see similar effects as with Aricept. I am not sure about the design; perhaps Lon has an idea about that?

Steve DeKosky: There are no data on efficacy. By that, I mean the type that might emerge from a controlled trial. 
June Kinoshita: Yes, efficacy data are difficult to obtain. In the meantime, people must also worry about potential adverse effects from combining two approved therapies.

Jim Kallio: So do we even know yet about synergistic effects of adding the cholinesterase inhibitor with memantine?

Steve DeKosky: I think there are many cases in which people have done that - added memantine to ongoing donepezil or galantamine. The former case would be like the Tariot study. I do not know if you can get an answer about "synergy" from that. Combined effects may depend on the stage at which you initiate the therapies.

Jim Kallio: A lot of caregiver concern is about whether it is worth the money to add memantine therapy ... It can be expensive without insurance.

Lon Schneider: Again, no evidence that memantine and donepezil together provide additive effects, but strong evidence that memantine provides effects in people who had been taking donepezil for an average of two years or so.

Dave Grillot: I think the big questions for families are: When should it be started and when is it no longer worthwhile, and apparently, should the person stay on cholinesterase inhibitors?

Steve DeKosky: Dave, specific data on which to make recommendations about your important questions are not available yet. No one knows when to take off the cholinesterase inhibitors. And although we know that the studies show effects of memantine on AD subjects with MMSE of 15 and below, we do not know "how high" to go - or will not with certainty until the mild to moderate AD studies are completed and reported, which we hope will be soon. The answer to when to stop is not a simple or unitary one, and requires careful discussion with your doctor and consideration of the status of the patient.

June Kinoshita: If a patient is already on, say, donepezil or Reminyl, can a physician feel comfortable about adding memantine to the treatment, in terms of potential adverse effects?

Lon Schneider: The evidence, as thin as it is, is that memantine added to donepezil reduces adverse effects, that it does not add AEs.
June Kinoshita: Lon, that is good to know. Based on what we know about the mechanism of memantine, are there any classes of drugs, foods, etc., that people should not combine with memantine?

Bengt Winblad: June, there are really few precautions to be taken, but certainly we have to be more careful with patients with reduced kidney function, recent heart infarction and, according to the recommendations, it should not be used in patients with epilepsy.

Susan Scruby: For what it is worth, my mother has been on Aricept for some time, though at a low dosage. She seems unable to tolerate the higher dosage (we have tried three times). She started on the titration pack of Namenda ${ }^{\circledR}$ on January 31 - it may just be wishful thinking on my part, but she does seem to be more engaged. Many of her conversations do not make sense, but she is more alert and aware, and more interested in talking with people and participating. This may pass, too, but I will take what I can get.

Bengt Winblad: Susan, I think what you report is exactly what caregivers report for us in Stockholm.

June Kinoshita: Sadly, our time is up. I want to thank our guests for participating in today's chat, and our audience for being here.

\section{References}

[1] P.N. Tariot, M.R. Farlow, G.T. Grossberg, S.M. Graham, S. McDonald and I. Gergel, Memantine treatment in patients with moderate to severe Alzheimer disease already receiving donepezil: a randomized controlled trial, JAMA 291 (2004), 21317-21324.

[2] A.H. Schapira and C.W. Olanow, Neuroprotection in Parkinson disease: mysteries, myths, and misconceptions, JAMA 291 (2004), 358-364.

[3] C.W. Ritchie, A.I. Bush, A. Mackinnon, S. Macfarlane, M. Mastwyk, L. MacGregor, L. Kiers, R. Cherny, Q.X. Li, A. Tammer, D. Carrington, C. Mavros, I. Volitakis, M. Xilinas, D. Ames, S. Davis, K. Beyreuther, R.E. Tanzi and C.L. Masters, Metalprotein attenuation with iodochlorhydroxyquin (clioquinol) targeting Abeta amyloid deposition and toxicity in Alzheimer disease: a pilot phase 2 clinical trial, Arch Neurol 60 (2003), 1685-1691.

[4] J.M. Orgogozo, A.S. Rigaud, A. Stöffler, H.J. Möbius and F. Forette, Efficacy and safety of memantine in patients with mild to moderate vascular dementia: A randomized, placebocontrolled trial (MMM 300), Stroke 33 (2002), 1834-1839.

[5] G. Wilcock, H.J. Möbius and A. Stöffler, A double-blind, placebo-controlled multicentre study of memantine in mild to moderate vascular dementia (MMM500), Int Clin Psychopharmacol 17 (2002), 297-305.

[6] B. Reisberg, R. Doody, A. Stöffler, F. Schmitt, S. Ferris and H.J. Möbius, Memantine in moderate-to-severe Alzheimer's disease, N Engl J Med 348 (2003), 1333-1341. 